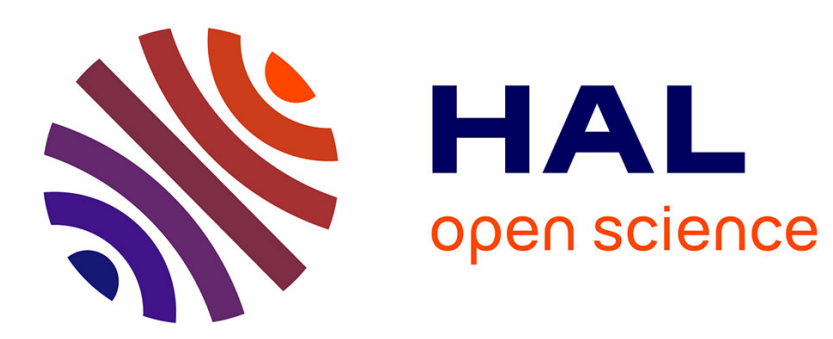

\title{
Hamiltonian reduced fluid model for plasmas with temperature and heat flux anisotropies
}

\author{
Emanuele Tassi
}

\section{To cite this version:}

Emanuele Tassi. Hamiltonian reduced fluid model for plasmas with temperature and heat flux anisotropies. Theoretical and Mathematical Physics, 2016, 188 (3), pp.1377 - 1393. 10.1134/S0040577916090099 . hal-01434164

\section{HAL Id: hal-01434164 \\ https://hal.science/hal-01434164}

Submitted on 13 Jan 2017

HAL is a multi-disciplinary open access archive for the deposit and dissemination of scientific research documents, whether they are published or not. The documents may come from teaching and research institutions in France or abroad, or from public or private research centers.
L'archive ouverte pluridisciplinaire HAL, est destinée au dépôt et à la diffusion de documents scientifiques de niveau recherche, publiés ou non, émanant des établissements d'enseignement et de recherche français ou étrangers, des laboratoires publics ou privés. 


\title{
Hamiltonian reduced fluid model for plasmas with temperature and heat flux anisotropies
}

\author{
E. Tassi ${ }^{1}$ \\ 1 Aix-Marseille Université, Université de Toulon, CNRS, \\ Centre de Physique Théorique, UMR 7332, 13288 Marseille, France
}

\begin{abstract}
We derive a Hamiltonian fluid model for strongly magnetized plasmas describing the evolution, for an arbitrary number of species, of density, velocity and electromagnetic fluctuations as well as of the temperature and heat flux fluctuations associated with motions parallel and perpendicular to the direction of a background magnetic field. The model is derived as a reduction of the infinite hierarchy of equations obtained by taking moments, with respect to Hermite-Laguerre polynomials in velocitymagnetic moment coordinates, of a Hamiltonian drift-kinetic system. A closure relation directly coupling the heat flux fluctuations in the directions parallel and perpendicular to the background magnetic field is shown to provide a fluid reduction which preserves the Hamiltonian character of the parent drift-kinetic model. An alternative set of dynamical variables is found, in terms of which the Poisson bracket of the fluid model takes a simple direct sum structure and permits an easy identification of the Casimir invariants. Such invariants, in the limit of translational symmetry with respect to the direction of the background magnetic field, turn out to be associated with Lagrangian invariants of the fluid model. It is pointed out that the coupling between parallel and perpendicular heat flux evolutions introduced by the closure, is necessary in order to guarantee the existence of a Hamiltonian structure with a Poisson bracket obtained as extension of a Lie-Poisson bracket.
\end{abstract}

\section{INTRODUCTION}

Kinetic theories provide an accurate description of continuous media such as gases and plasmas. Such models consist of evolution equations for distribution functions defined on particle phase space, possibly coupled self-consistently to equations governing the evolution of fields affecting the particle dynamics. Paradigmatic examples of kinetic theories are the Vlasov-Maxwell system, for plasmas in the presence of electromagnetic fields and the Vlasov-Poisson system, for plasmas in electrostatic fields, or for particle distributions subject to their own gravitational potential. A less accurate, but computationally less demanding, level of description of continuous media is provided by fluid models, which, in the Eulerian representation, consist of evolution equations for fluid dynamical variables depending only on spatial coordinates, such as particle densities, momenta, temperatures, etc..coupled with governing equations for the fields. The fluid dynamical variables usually correspond to moments of the kinetic distribution functions, obtained by integrating over velocity space the distribution functions, multiplied times non-negative integer powers of the velocity coordinates. Such powers determine the order of the moments.

Evolution equations for the fluid moments can then be derived directly from the evolution equations for the distribution functions. Because the evolution equation for each moment typically depends also on moments of higher order, this leads to an infinite hierarchy of coupled evolution equations for the fluid variables. In order to get a closed system for a finite number of fluid variables a reduction procedure, usually referred to as to closure, is required. Imposing a 
closure amounts to assume that a moment of a given order is determined by lower-order moments. Such lower-order moments become the dynamical variables of the fluid model which is then no longer equivalent to the original kinetic description but evolves only a finite number of dynamical variables depending only on the spatial coordinates.

Clearly, the choice of the closure relating a given moment to lower order moments, is crucial in determining the characteristics of the fluid model.

In the plasma physics literature several examples of closure relations exist, which satisfy different criteria. For instance in Refs. [1, 2] the closure is such to guarantee energy conservation for the resulting fluid model. Fluid models retaining kinetic effects such as Landau damping have been presented in Refs. [3-7]. Non-dissipative closures preserving time-reversal symmetry of the original kinetic equation have been proposed in Ref. [8]. A fluid closure based on entropy production arguments has been proposed in Ref. [9].

In spite of a considerable effort devoted to identifying fluid closures, little is known about the consequences of such closures on some structure of the model equations. In particular, in many cases it is not known whether, in the limit where the parent kinetic model has a Hamiltonian structure (which is the case for instance for Vlasov-Maxwell and Vlasov-Poisson systems $[10,11])$, the closure is such to preserve the Hamiltonian structure in the fluid model as well. This should be the case unless one voluntarily intends to introduce dissipation in the fluid model. In the mathematical physics literature, results related to this problem date back at least to Refs. [12, 13], where the connection between the Poisson bracket for the Vlasov equation and the Poisson brackets in terms of the moments had been elucidated. The connection between the Hamiltonian dynamics of the Vlasov moments and integrability has been discussed in Ref. [14]. The Hamiltonian structure of the Bogoliubov-Born-Green-Kirkwood-Yvon hierarchy of moment equations, on the other hand, had been presented in Ref. [15]. In Ref. [16] Hamiltonian structures for finite fluid reductions originating from the Vlasov-Poisson system are given. Some recent results in this context have also been obtained with regard to the identification of Hamiltonian fluid closures for three [17] and four-field [18] models obtained from the Vlasov-Poisson system, and two-field models obtained from drift-kinetic [19, 20] and gyrokinetic systems [21]. A closure relation, preserving a Hamiltonian structure in a fluid model derived from a drift-kinetic system and retaining an arbitrary number of moments, was presented in Ref. [22].

The results presented in this paper belong to this line of research, devoted to identifying closures that preserve the Hamiltonian structure of a parent Hamiltonian kinetic theory. In particular, in the present work, we consider, as parent kinetic theory, a system of drift-kinetic equations coupled with electromagnetic fields through a quasi-neutrality 
condition and Ampère's law. Drift-kinetic equations (see, e.g. Ref. [23]) are of interest for low-frequency phenomena in plasmas where an intense component of the magnetic field is present. Indeed, under these assumptions, the fast gyration motion of particles around the intense component of the magnetic field can be averaged out because it takes place on too short time scales, when compared to the frequencies of interest. As a result, when compared to the six-dimensional phase space of the Vlasov distribution function, the drift-kinetic equation possesses the advantage of being defined on a reduced five-dimensional phase space, where the gyration angle coordinate has been ignored because irrelevant for low-frequency phenomena. The Hamiltonian drift-kinetic model adopted in this paper can be derived from the gyrokinetic model treated in Ref. [2] upon applying a number of simplifying assumptions consisting of neglecting finite Larmor radius effects, background inhomogeneities and dissipative terms.

The purpose of this paper is to show that a closure relation exists so that it is possible to derive, from such Hamiltonian drift-kinetic system, a Hamiltonian fluid model evolving six field variables for each particle species. More precisely, indicating with $\mathcal{N}$ the number of particle species present in the plasma, we can construct a $6 \mathcal{N}$-field Hamiltonian fluid model evolving, for each species and self-consistently with the electromagnetic fields, the density, the canonical fluid momentum, temperature and heat flux fluctuations originated from both the parallel and perpendicular particle motions. Such field variables are proportional to moments of a generalized distribution function taken with respect to Hermite and Laguerre polynomials of normalized velocity along the strong component of the magnetic field and of magnetic moment, respectively.

With respect to recent related results, such as those of Refs. [19-21, 24], this model indeed accounts additionally also for moments taken with respect to the particle magnetic moment, thus yielding a fluid model that can describe also phenomena related to temperature and heat flux anisotropies.

The existence of the Hamiltonian structure in the resulting fluid model, besides assuring the absence of spurious dissipative effects and an unambiguous identification of the conserved total energy, can provide also information about further conservation laws of the system. Indeed, as is the case for the Hamiltonian structure of continuous media in the Eulerian description, also such fluid model turns out to possess a Poisson bracket of noncanonical type (see, e.g. Refs. [25, 26]). Poisson brackets of this type are characterized by the presence of Casimir invariants, which constrain the dynamics of the system. Thanks to the Hamiltonian structure, we also provide Casimir invariants of the model, which become particularly relevant in the two-dimensional limit, when the system is translationally invariant with respect to the direction of the intense component of the magnetic field. In this limit, infinite families of Casimir 
invariants emerge. The identification of the Casimir invariants is made possible thanks to a change of variables in terms of which we are able to cast the Poisson bracket of the model in its simplest form, unveiling its direct sum structure. This alternative set of variables, which are referred to as to normal fields, turns out to be related to the existence of $6 \mathcal{N}$ Lagrangian invariants for the model in its two-dimensional limit.

The paper is organized as follows. In Sec. II the parent drift-kinetic model is introduced and its Hamiltonian structure is presented. In Sec. III we introduce the hierarchy of fluid equations, in terms of moments with respect to Hermite-Laguerre polynomials, and present the closure relation. The Hamiltonian structure of the resulting fluid model is presented in Sec. IV, whereas in Sec. V we introduce the normal field variables and present the Casimir invariants. We conclude in Sec. VI and provide the A where the eigenvalues of the matrix $\mathcal{W}^{(1)}{ }_{s}$ are explicitly determined.

\section{HAMILTONIAN PARENT DRIFT-KINETIC MODEL}

In a Cartesian coordinate system $(x, y, z)$ we consider the following system of drift-kinetic evolution equations :

$$
\frac{\partial g_{s}}{\partial t}+\frac{c}{B}\left[\phi-\frac{v}{c} A, g_{s}\right]+v \frac{\partial}{\partial z}\left(g_{s}+q_{s} \frac{\mathcal{F}_{s}}{T_{s}}\left(\phi-\frac{v}{c} A\right)\right)=0
$$

complemented by the quasi-neutrality relation

$$
\frac{n_{0} M c^{2}}{B^{2}} \Delta_{\perp} \phi=-\sum_{s} q_{s} \int d \mathcal{W}_{s} g_{s}
$$

and by Ampère's law

$$
\frac{c}{4 \pi} \Delta_{\perp} A-\sum_{s} \frac{q_{s}^{2} n_{0}}{m_{s} c} A=-\sum_{s} q_{s} \int d \mathcal{W}_{s} v g_{s}
$$

The index $s=1, \cdots, \mathcal{N}$ labels the $\mathcal{N}$ particle species present in the plasma and, in order to simplify the notation, the sum over $s$ always means that the index $s$ goes from 1 to $\mathcal{N}$. The dynamical field variable $g_{s}$ is defined as

$$
g_{s}\left(x, y, z, v, \mu_{s}, t\right)=\tilde{f}_{s}\left(x, y, z, v, \mu_{s}, t\right)+\frac{q_{s}}{T_{s}} \frac{v}{c} \mathcal{F}_{s}\left(v, \mu_{s}\right) A(x, y, z, t)
$$

where $\tilde{f}_{s}$ is the perturbation of the distribution function for the $s$-th species with respect to the equilibrium Maxwellian

$$
\mathcal{F}_{s}\left(v, \mu_{s}\right)=n_{0}\left(\frac{m_{s}}{2 \pi T_{s}}\right)^{3 / 2} \exp \left(-\frac{v^{2}}{2 v_{t s}^{2}}-\frac{\mu_{s} B}{m_{s} v_{t s}^{2}}\right)
$$

with $v$ indicating the velocity coordinate along $z$, which is the direction of the magnetic guide field, with $\mu_{s}=$ $m_{s}\left(v_{x}^{2}+v_{y}^{2}\right) /(2 B)$ indicating the magnetic moment of $s$-th particle species and with the constants $q_{s}, m_{s}, T_{s}, v_{t s}$ 
$c$ and $n_{0}$ indicating the particle charge and mass, the equilibrium temperature for the $s$-th species, the thermal speed $v_{t s}=\sqrt{T_{s} / m_{s}}$, the speed of light and the equilibrium density, respectively. The magnetic field is given by $\mathbf{B}(x, y, z, t)=\nabla A(x, y, z, t) \times \hat{z}+B \hat{z}$, with constant $B$, whereas $\phi=\phi(x, y, z, t)$ indicates the electrostatic potential. In Eq. (2) the constant $M$ is defined as $M=\sum_{s} m_{s}$. We indicated with the symbol $\Delta_{\perp}$ the perpendicular Laplacian operator $\Delta_{\perp}=\partial_{x x}+\partial_{y y}$, whereas the bracket $[$,$] is defined as [f, g]=\partial_{x} f \partial_{y} g-\partial_{y} f \partial_{x} g$, for two functions $f$ and $g$. Finally, the volume element $d \mathcal{W}_{s}$ is defined as $d \mathcal{W}_{s}=2 \pi\left(B / m_{s}\right) d \mu_{s} d v$. The spatial coordinates $x, y$ and $z$ vary over the 3 -dimensional torus $\mathbb{T}^{3}$, whereas $v \in \mathbb{R}, \mu_{s} \geq 0$ for all $s$ and $t \geq 0$. The perturbations of the distribution functions $\tilde{f}_{s}$ are assumed to decay to zero sufficiently rapidly as $v \rightarrow \pm \infty$ so that boundary terms vanish when integrating by parts with respect to $v$. The electrostatic potential $\phi$ is also assumed to have zero mean value, so that the Laplacian operator can be inverted in Eq. (2).

The model consisting of Eqs. (1), (2) and (3) can be derived from the gyrokinetic model described for instance in Ref. [2], by taking the limit of vanishing Larmor radius, and in the absence of background magnetic inhomogeneities and dissipation. The model assumes the common $\delta f$ approximation which is valid for plasmas close to equilibrium, i.e. $\tilde{f}_{s} \ll \mathcal{F}_{s}$ and with weak variations along the guide field, i.e. $\partial / \partial z \ll \partial / \partial x$ and $\partial / \partial z \ll \partial / \partial y$. With regard to Eq. (2) we observe that, with respect to the quasi-neutrality relation adopted in Ref. [2], the finite Larmor radius contribution depending on the perpendicular temperature has been neglected, whereas the leading order contribution polarization term has been retained. A concrete example where this assumption is justified corresponds to the case where two species are present in the plasma, with electrons retaining temperature and heat flux anisotropies, and ions following an isothermal description.

The parent drift-kinetic model (1), (2) and (3) possesses a Hamiltonian structure which is a straighforward extension of the Hamiltonian structure of the model treated in Ref. [27]. Such Hamiltonian structure consists of the Hamiltonian functional

$$
H\left(g_{1}, \cdots, g_{\mathcal{N}}\right)=\sum_{s} \frac{1}{2} \int d^{3} x d \mathcal{W}_{s}\left[T_{s} \frac{g_{s}^{2}}{\mathcal{F}_{s}}+q_{s} g_{s}\left(\phi-\frac{v}{c} A\right)\right]
$$

and of the noncanonical Poisson bracket

$$
\{F, G\}=\sum_{s}\{F, G\}_{s}=-\sum_{s} \int d^{3} x d \mathcal{W}_{s}\left(\frac{c}{q_{s} B} g_{s}\left[F_{g_{s}}, G_{g_{s}}\right]+v \frac{\mathcal{F}_{s}}{T_{s}} F_{g_{s}} \frac{\partial G_{g_{s}}}{\partial z}\right)
$$

where the subscripts on the functionals $F$ and $G$ indicates functional derivatives. Indeed, the generic expression for 
a Hamiltonian system describing the dynamics of $N$ field variables $\chi_{1}, \cdots, \chi_{N}$ is given by

$$
\frac{\partial \chi_{i}}{\partial t}=\left\{\chi_{i}, H\right\}, \quad i=1, \cdots, N
$$

where $H=H\left(\chi_{1}, \cdots, \chi_{N}\right)$ is the Hamiltonian functional and $\{$,$\} a Poisson bracket. One obtains that, with N=\mathcal{N}$, by substituting $\chi_{1}, \cdots, \chi_{\mathcal{N}}$ with $g_{1}, \cdots, g_{\mathcal{N}}, H$ with the Hamiltonian functional (6) and using as Poisson bracket the one indicated in Eq. (7), yields namely the drift-kinetic evolution equations (1).

We remark that, whereas the Poisson bracket (7) consists of the direct sum of Poisson brackets each of which is associated with a given species, the coupling between the different species in the dynamics comes from the last two terms in the Hamiltonian (6), containing $\phi$ and $A$, which involve all species according to (2) and (3), respectively. The first term in the Hamiltonian (6), on the other hand, comes from expanding the free energy functional of the original full (i.e. without the $\delta f$ approximation) drift-kinetic model up to second order, about the equilibrium Maxwellian $(5)$.

\section{FLUID EQUATIONS}

From the drift-kinetic equations (1) a hierarchy of evolution equations for dynamical variables depending only on the spatial coordinates $x, y, z$, as well as on time, can be obtained. As dynamical variables for a given species $s$, we choose the moments $g_{m n_{s}}$ defined as

$$
g_{m n_{s}}(x, y, z, t)=\frac{1}{n_{0} \sqrt{m !}} \int d \mathcal{W}_{s} H_{m}\left(\frac{v}{v_{t s}}\right) L_{n}\left(\frac{\mu_{s} B}{m_{s} v_{t s}^{2}}\right) g_{s}\left(x, y, z, v, \mu_{s}, t\right)
$$

where $H_{m}$ and $L_{n}$ are the Hermite and Laguerre polynomials of order $m$ and $n$, respectively, with $m$ and $n$ non-negative integers. We recall that such polynomials can be defined in the following way

$$
H_{m}(x)=(-1)^{m} \mathrm{e}^{x^{2} / 2} \frac{d^{m}}{d x^{m}} \mathrm{e}^{-x^{2} / 2}, \quad L_{n}(x)=\frac{\mathrm{e}^{x}}{n !} \frac{d^{n}}{d x^{n}}\left(x^{n} \mathrm{e}^{-x}\right)
$$

The first Hermite and Laguerre polynomials, which correspond in particular to those of interest for the present analysis, are then given by

$$
\begin{aligned}
& H_{0}\left(\frac{v}{v_{t s}}\right)=1, \quad H_{1}\left(\frac{v}{v_{t s}}\right)=\frac{v}{v_{t s}}, \quad H_{2}\left(\frac{v}{v_{t s}}\right)=\frac{v^{2}}{v_{t s}^{2}}-1, \\
& H_{3}\left(\frac{v}{v_{t s}}\right)=\frac{v^{3}}{v_{t s}^{3}}-3 \frac{v}{v_{t s}}, \quad L_{0}\left(\frac{\mu_{s} B}{m_{s} v_{t s}^{2}}\right)=1, \quad L_{1}\left(\frac{\mu_{s} B}{m_{s} v_{t s}^{2}}\right)=1-\frac{\mu_{s} B}{m_{s} v_{t s}^{2}} .
\end{aligned}
$$


The choice of the Hermite and Laguerre polynomials as "weight" functions, in Eq. (9), for the parallel velocity and magnetic moments, respectively, turns out to be practical due to the orthogonality relation

$$
\int d \mathcal{W}_{s} H_{m}\left(\frac{v}{v_{t s}}\right) H_{i}\left(\frac{v}{v_{t s}}\right) L_{n}\left(\frac{\mu_{s} B}{m_{s} v_{t s}^{2}}\right) L_{j}\left(\frac{\mu_{s} B}{m_{s} v_{t s}^{2}}\right) \mathcal{F}_{s}=n_{0} m ! \delta_{m i} \delta_{n j}
$$

which involves the equilibrium Maxwellian $\mathcal{F}_{s}$. The dynamical variable $g_{s}$ is decomposed over the basis of Hermite and Laguerre polynomials in the following way

$$
g_{s}\left(x, y, z, v, \mu_{s}, t\right)=\sum_{m, n=0}^{+\infty} \frac{1}{\sqrt{m !}} H_{m}\left(\frac{v}{v_{t s}}\right) L_{n}\left(\frac{\mu_{s} B}{m_{s} v_{t s}^{2}}\right) \mathcal{F}_{s}\left(v, \mu_{s}\right) g_{m n_{s}}(x, y, z, t)
$$

from which the expression (9) follows by making use of the relation (12).

We consider here the evolution of the moments $g_{00_{s}}, g_{10_{s}}, g_{20_{s}}, g_{30 s}, g_{01_{s}}$ and $g_{11_{s}}$. Such moments are indeed also proportional to the physically most relevant dynamical variables typically adopted in anisotropic reduced fluid models for plasmas, in the presence of a strong guide field. The correspondence between the moments and the physical variables is given by :

$$
\begin{aligned}
& g_{00_{s}}=\frac{n_{s}}{n_{0}}, \quad g_{10 s}=\frac{u_{s}}{v_{t s}}+q_{s} \frac{A}{m_{s} v_{t s} c}, \quad g_{20_{s}}=\frac{1}{\sqrt{2}} \frac{T_{\|_{s}}}{T_{s}}, \\
& g_{30 s}=\sqrt{\frac{2}{3}} \frac{1}{n_{0}} \frac{q_{\|_{s}}}{T_{s} v_{t s}}, \quad g_{01 s}=-\frac{T_{\perp s}}{T_{s}}, \quad g_{11_{s}}=-\frac{1}{n_{0}} \frac{q_{\perp s}}{T_{s} v_{t s}},
\end{aligned}
$$

where, for each species $s, n_{s}=\int d \mathcal{W}_{s} \tilde{f}_{s}$ are the density fluctuations, $u_{s}=\left(1 / n_{0}\right) \int d \mathcal{W}_{s} v \tilde{f}_{s}$ the velocity fluctuations, $T_{\|_{s}}=\left(1 / n_{0}\right) \int d \mathcal{W}_{s}\left(m_{s} v^{2}-T_{s}\right) \tilde{f}_{s}$ and $T_{\perp s}=\left(1 / n_{0}\right) \int d \mathcal{W}_{s}\left(\mu_{s} B-T_{s}\right) \tilde{f}_{s}$ the parallel and perpendicular temperature fluctuations, respectively, whereas $q_{\|_{s}}=(1 / 2) \int d \mathcal{W}_{s}\left(m_{s} v^{2}-3 T_{s}\right) v \tilde{f}_{s}$ and $q_{\perp s}=\int d \mathcal{W}_{s}\left(\mu_{s} B-T_{s}\right) v \tilde{f}_{s}$ are the parallel and perpendicular heat flux fluctuations, respectively.

By multiplying both sides of Eq. (1) for $H_{m}\left(v / v_{t s}\right) L_{n}\left(\mu_{s} B /\left(m_{s} v_{t s}^{2}\right)\right)$ and integrating over $d \mathcal{W}_{s}$ one obtains, for the 
six moments indicated in Eq. (14), the following set of evolution equations:

$$
\begin{aligned}
& \frac{\partial g_{00_{s}}}{\partial t}=-\frac{c}{B}\left[\phi, g_{00_{s}}\right]+\frac{v_{t s}}{B}\left[A, g_{10_{s}}\right]-v_{t s} \frac{\partial g_{10_{s}}}{\partial z}+v_{t s}^{2} \frac{q_{s}}{c T_{s}} \frac{\partial A}{\partial z}, \\
& \frac{\partial g_{10 s}}{\partial t}=-\frac{c}{B}\left[\phi, g_{10_{s}}\right]+\sqrt{2} \frac{v_{t s}}{B}\left[A, g_{20 s}\right]+\frac{v_{t s}}{B}\left[A, g_{00 s}\right] \\
& -\sqrt{2} v_{t s} \frac{\partial g_{20} s}{\partial z}-v_{t s} \frac{\partial g_{002}}{\partial z}-v_{t s} \frac{q_{s}}{T_{s}} \frac{\partial \phi}{\partial z}, \\
& \frac{\partial g_{20 s}}{\partial t}=-\frac{c}{B}\left[\phi, g_{20 s}\right]+\sqrt{3} \frac{v_{t s}}{B}\left[A, g_{30 s}\right]+\sqrt{2} \frac{v_{t s}}{B}\left[A, g_{10_{s}}\right] \\
& -\sqrt{3} v_{t s} \frac{\partial g_{30}}{\partial z}-\sqrt{2} v_{t s} \frac{\partial g_{10_{s}}}{\partial z}+\sqrt{2} v_{t s}^{2} \frac{q_{s}}{c T_{s}} \frac{\partial A}{\partial z}, \\
& \frac{\partial g_{30}}{\partial t}=-\frac{c}{B}\left[\phi, g_{30 s}\right]+2 \frac{v_{t s}}{B}\left[A, g_{40 s}\right]+\sqrt{3} \frac{v_{t s}}{B}\left[A, g_{20 s}\right] \\
& -2 v_{t s} \frac{\partial g_{40 s}}{\partial z}-\sqrt{3} v_{t s} \frac{\partial g_{20}}{\partial z} \\
& \frac{\partial g_{01 s}}{\partial t}=-\frac{c}{B}\left[\phi, g_{01_{s}}\right]+\frac{v_{t s}}{B}\left[A, g_{11_{s}}\right]-v_{t s} \frac{\partial g_{11_{s}}}{\partial z}, \\
& \frac{\partial g_{11_{s}}}{\partial t}=-\frac{c}{B}\left[\phi, g_{11_{s}}\right]+\sqrt{2} \frac{v_{t s}}{B}\left[A, g_{21_{s}}\right]+\frac{v_{t s}}{B}\left[A, g_{01 s}\right] \\
& -\sqrt{2} v_{t s} \frac{\partial g_{21_{s}}}{\partial z}-v_{t s} \frac{\partial g_{01 s}}{\partial z},
\end{aligned}
$$

where use of the recursive relation $H_{m+1}(x)=x H_{m}(x)-m H_{m-1}(x)$ has been made.

From Eqs. (18) and (20) it emerges that the system is not closed, because such two equations depend on $g_{40} s$ and $g_{21_{s}}$, which are undetermined. This leads to a closure problem, amounting to express $g_{40}$ and $g_{21_{s}}$ in terms of the six lower order moments. Recalling that we require to preserve, in the fluid model, the Hamiltonian character of the parent drift-kinetic model, we intend to show that closing the system (15)-(20) by imposing the relations

$$
g_{40 s}=\alpha_{s} g_{11_{s}}, \quad g_{21_{s}}=\sqrt{2} \alpha_{s} g_{30 s}
$$

with non-vanishing constant $\alpha_{s}$, leads to a fluid model with a Hamiltonian structure.

\section{HAMILTONIAN ANISOTROPIC FLUID MODEL}

In order to simplify the notation we rename the moment variables in the following way:

$$
\begin{array}{ll}
g_{0 s}=g_{00 s}, & g_{1 s}=g_{10 s}, \quad g_{2 s}=g_{20 s}, \\
g_{3 s}=g_{30 s}, & g_{4 s}=g_{01_{s}}, \quad g_{5 s}=g_{11_{s}} .
\end{array}
$$


Applying the closure relation (21) we obtain that the anisotropic fluid model reads

$$
\begin{aligned}
\frac{\partial g_{0_{s}}}{\partial t}= & =-\frac{c}{B}\left[\phi, g_{0_{s}}\right]+\frac{v_{t s}}{B}\left[A, g_{1_{s}}\right]-v_{t s} \frac{\partial g_{1_{s}}}{\partial z}+v_{t s}^{2} \frac{q_{s}}{c T_{s}} \frac{\partial A}{\partial z} \\
\frac{\partial g_{1_{s}}}{\partial t} & =-\frac{c}{B}\left[\phi, g_{1_{s}}\right]+\sqrt{2} \frac{v_{t s}}{B}\left[A, g_{2_{s}}\right]+\frac{v_{t s}}{B}\left[A, g_{0 s}\right] \\
& -\sqrt{2} v_{t s} \frac{\partial g_{2_{s}}}{\partial z}-v_{t s} \frac{\partial g_{0_{s}}}{\partial z}-v_{t s} \frac{q_{s}}{T_{s}} \frac{\partial \phi}{\partial z} \\
\frac{\partial g_{2_{s}}}{\partial t} & =-\frac{c}{B}\left[\phi, g_{2_{s}}\right]+\sqrt{3} \frac{v_{t s}}{B}\left[A, g_{3_{s}}\right]+\sqrt{2} \frac{v_{t s}}{B}\left[A, g_{1_{s}}\right] \\
& -\sqrt{3} v_{t s} \frac{\partial g_{3_{s}}}{\partial z}-\sqrt{2} v_{t s} \frac{\partial g_{1_{s}}}{\partial z}+\sqrt{2} v_{t s}^{2} \frac{q_{s}}{c T_{s}} \frac{\partial A}{\partial z} \\
\frac{\partial g_{3_{s}}}{\partial t} & =-\frac{c}{B}\left[\phi, g_{3_{s}}\right]+2 \alpha_{s} \frac{v_{t s}}{B}\left[A, g_{5 s}\right]+\sqrt{3} \frac{v_{t s}}{B}\left[A, g_{2 s}\right] \\
& -2 \alpha_{s} v_{t s} \frac{\partial g_{5_{s}}}{\partial z}-\sqrt{3} v_{t s} \frac{\partial g_{2_{s}}}{\partial z} \\
\frac{\partial g_{4_{s}}}{\partial t} & =-\frac{c}{B}\left[\phi, g_{4_{s}}\right]+\frac{v_{t s}}{B}\left[A, g_{5_{s}}\right]-v_{t s} \frac{\partial g_{5_{s}}}{\partial z} \\
\frac{\partial g_{5_{s}}}{\partial t} & =-\frac{c}{B}\left[\phi, g_{5_{s}}\right]+2 \alpha_{s} \frac{v_{t s}}{B}\left[A, g_{3 s}\right]+\frac{v_{t s}}{B}\left[A, g_{4 s}\right] \\
& -2 \alpha_{s} v_{t s} \frac{\partial g_{3_{s}}}{\partial z}-v_{t s} \frac{\partial g_{4_{s}}}{\partial z}
\end{aligned}
$$

In order to show that the system (22)-(27) possesses a Hamiltonian structure, we need to show that it can be cast in the form (8). A natural choice for the $6 \mathcal{N}$ field variables is clearly given by $\chi_{i_{s}}=g_{i_{s}}$, with $i=0, \cdots, 5$ and $s$ labelling the $\mathcal{N}$ particle species.

A natural candidate as Hamiltonian functional is obtained directly from the expression of the Hamiltonian of the parent model (6) upon replacing $g_{s}$ with the following truncated version of the series (13):

$$
\begin{aligned}
& g_{s}\left(x, y, z, v, \mu_{s}, t\right)=\mathcal{F}_{s}\left(v, \mu_{s}\right)\left[g_{0 s}(x, y, z, t) H_{0}\left(\frac{v}{v_{t s}}\right) L_{0}\left(\frac{\mu_{s} B}{m_{s} v_{t s}^{2}}\right)\right. \\
& +g_{1_{s}}(x, y, z, t) H_{1}\left(\frac{v}{v_{t s}}\right) L_{0}\left(\frac{\mu_{s} B}{m_{s} v_{t s}^{2}}\right)+\frac{g_{2 s}}{\sqrt{2}}(x, y, z, t) H_{2}\left(\frac{v}{v_{t s}}\right) L_{0}\left(\frac{\mu_{s} B}{m_{s} v_{t s}^{2}}\right) \\
& +\frac{g_{3 s}}{\sqrt{6}}(x, y, z, t) H_{3}\left(\frac{v}{v_{t s}}\right) L_{0}\left(\frac{\mu_{s} B}{m_{s} v_{t s}^{2}}\right)+g_{4 s}(x, y, z, t) H_{0}\left(\frac{v}{v_{t s}}\right) L_{1}\left(\frac{\mu_{s} B}{m_{s} v_{t s}^{2}}\right) \\
& \left.+g_{5 s}(x, y, z, t) H_{1}\left(\frac{v}{v_{t s}}\right) L_{1}\left(\frac{\mu_{s} B}{m_{s} v_{t s}^{2}}\right)\right],
\end{aligned}
$$

which retains only the contribution coming from the moments involved in the model. By inserting the truncated series (28) into the drift-kinetic Hamiltonian (6) one obtains, with the help of the orthogonality relation (12),

$$
H\left(g_{01}, \cdots, g_{5 \mathcal{N}}\right)=\frac{1}{2} \sum_{s} \int d^{3} x\left[n_{0} T_{s} \sum_{i=0}^{5} g_{i s}^{2}+q_{s} n_{0} g_{0 s} \phi-q_{s} n_{0} \frac{v_{t s}}{c} g_{1_{s}} A\right] .
$$

The functional (29) is a conserved quantity for the system (22)-(27) and can therefore be considered as a candidate Hamiltonian. This functional corresponds, in the appropriate limit, to the total conserved energy functional of the gyrofluid model of Ref. [2]. 
Concerning the Poisson bracket, the form of the model equations suggests that it be of the form $\{F, G\}=\sum_{s}\{F, G\}_{s}$, with

$$
\{F, G\}_{s}=-\frac{c}{q_{s} B n_{0}} \sum_{i, j, k=0}^{5} W_{k s}^{i j} \int d^{3} x g_{k_{s}}\left[F_{i_{s}}, G_{j_{s}}\right]-\frac{v_{t s}}{n_{0} T_{s}} \sum_{i, j=0}^{5} \mathcal{A}^{i j}{ }_{s} \int d^{3} x F_{i_{s}} \frac{\partial G_{j_{s}}}{\partial z} .
$$

In Eq. (30), $W_{k s}^{i j}$ and $\mathcal{A}^{i j}{ }_{s}$ are constants and the subscripts on functionals indicate functional derivatives, so that, for instance, $F_{i_{s}}=\delta F / \delta g_{i_{s}}$. Poisson brackets of the form (30) occur very often in Hamiltonian reduced fluid models for plasmas, assuming the presence of a strong magnetic guide field. Examples of such models can be found in Refs. [28-36]. In order to determine the coefficients $W_{k s}^{i j}$ and $\mathcal{A}^{i j}{ }_{s}$ identifying the Poisson bracket, we proceed in the following way. First, we consider the case $\mathcal{A}^{i j}{ }_{s}=0$, for all $i, j=0, \cdots, 5$ and look for coefficients $W_{k s}^{i j}$ such that the operation

$$
\{F, G\}_{\perp_{s}}=-\frac{c}{q_{s} B n_{0}} \sum_{i, j, k=0}^{5} W_{k s}^{i j} \int d^{3} x g_{k_{s}}\left[F_{i s}, G_{j_{s}}\right]
$$

is a Poisson bracket of its own and that, when combined with the candidate Hamiltonian (29), it yields the model equations (22)-(27) in the "perpendicular" limit, i.e. when all the terms involving $z$-derivatives are neglected. Subsequently, inspired by the procedure described in Ref. [29], we identify the coefficients $\mathcal{A}^{i j}{ }_{s}$ such that the bracket (30), again combined with the functional (29), yields the full model equations, and verify that the resulting bracket is a Poisson bracket.

To carry out the first part of the procedure, we recall, from Ref. [37], that the bilinear operation (31) is a Poisson bracket if and only if the matrices $\mathcal{W}^{(j)}{ }_{s}$, defined as

$$
\left(\mathcal{W}_{s}^{(j)}\right)^{k i}=W_{k s}^{i j}, \quad i, j, k=0, \cdots, 5
$$

with rows labelled by $k$ and and columns by $i$, are symmetric in the upper indices, i.e. $\left(\mathcal{W}^{(j)} s\right)^{k i}=\left(\mathcal{W}^{(j)} s\right)^{i k}$, and they all pairwise commute. Such two properties indeed are equivalent to the antisymmetry and the Jacobi identity, respectively, for the bracket (31). We remark that the Leibniz identity is automatically satisfied by a bracket of the form (31). In addition to the constraints related to the properties of a Poisson bracket, we require the coefficients $W_{k s}^{i j}$ (or, equivalently, the matrices $\mathcal{W}^{(j)} s$ ) to be such that the Hamiltonian evolution equations

$$
\frac{\partial g_{l_{s}}}{\partial t}=\left\{g_{l_{s}}, H\right\}_{\perp_{s}}=-\frac{c}{q_{s} B n_{0}} \sum_{j, k=0}^{5} W_{k s}^{l j}\left[H_{j_{s}}, g_{k_{s}}\right], \quad l=0, \cdots, 5
$$

correspond, when using $H$ given by Eq. (29), to the model Eqs. (22)-(27) in the limit when terms involving $z$ derivatives are set to zero. To this scope, it is useful to note that the functional derivatives of the Hamiltonian (29) 
are given by

$$
H_{0 s}=n_{0} T_{s} g_{0_{s}}+q_{s} n_{0} \phi, \quad H_{1 s}=n_{0} T_{s} g_{1_{s}}-q_{s} n_{0} \frac{v_{t s}}{c} A, \quad H_{i_{s}}=n_{0} T_{s} g_{i_{s}}, \quad i=2, \cdots, 5 .
$$

With this information, one finds that, in order for Eqs. (33) to match Eqs. (22)-(27), and for the bracket (31) to be antisymmetric, the matrices $\mathcal{W}^{(j)}{ }_{s}$ must be of the form

$$
\begin{aligned}
& \mathcal{W}_{s}^{(0)}=\left(\begin{array}{cccccc}
1 & 0 & 0 & 0 & 0 & 0 \\
0 & 1 & 0 & 0 & 0 & 0 \\
0 & 0 & 1 & 0 & 0 & 0 \\
0 & 0 & 0 & 1 & 0 & 0 \\
0 & 0 & 0 & 0 & 1 & 0 \\
0 & 0 & 0 & 0 & 0 & 1
\end{array}\right), \quad \mathcal{W}_{s}^{(1)}=\left(\begin{array}{cccccc}
0 & 1 & 0 & 0 & 0 & 0 \\
1 & 0 & \sqrt{2} & 0 & 0 & 0 \\
0 & \sqrt{2} & 0 & \sqrt{3} & 0 & 0 \\
0 & 0 & \sqrt{3} & 0 & 0 & 2 \alpha_{s} \\
0 & 0 & 0 & 0 & 0 & 1 \\
0 & 0 & 0 & 2 \alpha_{s} & 1 & 0
\end{array}\right), \\
& \mathcal{W}_{s}^{(2)}=\left(\begin{array}{cccccc}
0 & 0 & 1 & 0 & 0 & 0 \\
0 & \sqrt{2} & 0 & \sqrt{3} & 0 & 0 \\
1 & 0 & a_{1 s} & b_{1 s} & b_{2 s} & b_{3 s} \\
0 & \sqrt{3} & b_{1 s} & b_{4 s} & c_{1 s} & c_{2 s} \\
0 & 0 & b_{2 s} & c_{1 s} & b_{7 s} & c_{3 s} \\
0 & 0 & b_{3 s} & c_{2 s} & c_{3 s} & b_{10 s}
\end{array}\right), \quad \mathcal{W}^{(3)}{ }_{s}=\left(\begin{array}{cccccc}
0 & 0 & 0 & 1 & 0 & 0 \\
0 & 0 & \sqrt{3} & 0 & 0 & 2 \alpha_{s} \\
0 & \sqrt{3} & b_{1 s} & b_{4 s} & c_{1 s} & c_{2 s} \\
1 & 0 & b_{4 s} & a_{2 s} & b_{5 s} & b_{6 s} \\
0 & 0 & c_{1 s} & b_{5 s} & b_{8 s} & c_{4 s} \\
0 & 2 \alpha_{s} & c_{2 s} & b_{6 s} & c_{4 s} & b_{11 s}
\end{array}\right), \\
& \mathcal{W}_{s}^{(4)}=\left(\begin{array}{cccccc}
0 & 0 & 0 & 0 & 1 & 0 \\
0 & 0 & 0 & 0 & 0 & 1 \\
0 & 0 & b_{2 s} & c_{1 s} & b_{7 s} & c_{3 s} \\
0 & 0 & c_{1 s} & b_{5 s} & b_{8 s} & c_{4 s} \\
1 & 0 & b_{7 s} & b_{8 s} & a_{3 s} & b_{9 s} \\
0 & 1 & c_{3 s} & c_{4 s} & b_{9 s} & b_{12 s}
\end{array}\right), \quad \mathcal{W}^{(5)}{ }_{s}=\left(\begin{array}{cccccc}
0 & 0 & 0 & 0 & 0 & 1 \\
0 & 0 & 0 & 2 \alpha_{s} & 1 & 0 \\
0 & 0 & b_{3 s} & c_{2 s} & c_{3 s} & b_{10 s} \\
0 & 2 \alpha_{s} & c_{2 s} & b_{6 s} & c_{4 s} & b_{11 s} \\
0 & 1 & c_{3 s} & c_{4 s} & b_{9 s} & b_{12 s} \\
1 & 0 & b_{10 s} & b_{11 s} & b_{12 s} & a_{4 s}
\end{array}\right),
\end{aligned}
$$

where $a_{1 s}, \cdots, a_{4 s}, b_{1 s}, \cdots, b_{12 s}, c_{1 s}, \cdots, c_{4 s}$ are constants to be determined by the constraint of the Jacobi identity. Indeed we can observe that there exist three types of contributions that can be added to the bracket (31) in such a way that antisymmetry is respected and that, adopting (29) as Hamiltonian, the equations of motion do not get 
modified. These three types of terms correspond to

$$
\begin{array}{r}
\int d^{3} x g_{i_{s}}\left[F_{i_{s}}, G_{i_{s}}\right], \quad i \in\{2,3,4,5\} \\
\int d^{3} x\left[g_{i_{s}}\left[F_{j_{s}}, G_{j_{s}}\right]+g_{j_{s}}\left(\left[F_{i_{s}}, G_{j_{s}}\right]+\left[F_{j_{s}}, G_{i_{s}}\right]\right)\right], \\
i, j \in\{2,3,4,5\}, \quad i \neq j \\
\int d^{3} x\left[g_{i_{s}}\left(\left[F_{j_{s}}, G_{k_{s}}\right]+\left[F_{k_{s}}, G_{j_{s}}\right]\right)+g_{j_{s}}\left(\left[F_{k_{s}}, G_{i_{s}}\right]+\left[F_{i_{s}}, G_{k_{s}}\right]\right)\right. \\
\left.+g_{k_{s}}\left(\left[F_{i_{s}}, G_{j_{s}}\right]+\left[F_{j_{s}}, G_{i_{s}}\right]\right)\right], \quad i, j, k \in\{2,3,4,5\}, \quad i \neq j \neq k
\end{array}
$$

Because $H_{i_{s}}=n_{0} T_{s} g_{i_{s}}$, for $i=2, \cdots, 5$, it is not difficult to see that any linear combination of terms of the type (36), (37) and (38), when included in the bracket, is irrelevant for the equations of motion $(33)$ given that $\left[g_{i_{s}}, g_{i_{s}}\right]=0$. Such terms, however, although transparent for the model dynamics, can be crucial for the Jacobi identity.

The matrices (35) account for the most general linear combinations of terms of the type (36)-(38). In particular, $a_{1 s}, \cdots, a_{4 s}$ are coefficients of the terms of the type (36), whereas $b_{1 s}, \cdots, b_{12 s}$ and $c_{1 s}, \cdots, c_{4 s}$ are coefficients of terms of the type (37) and (38), respectively.

As above mentioned, the Jacobi identity for the bracket (31), is satisfied if and only if all the matrices in Eq. (35) pairwise commute. Imposing this condition determines the arbitrary coefficients as follows:

$$
\begin{aligned}
& a_{1 s}=2 \sqrt{2}, \quad a_{2 s}=0, \quad a_{3 s}=0, \quad a_{4 s}=-\frac{1+2 \alpha_{s}^{2}-8 \alpha_{s}^{4}}{\sqrt{6} \alpha_{s}}, \\
& b_{1 s}=0, \quad b_{2 s}=0, \quad b_{3 s}=\sqrt{6} \alpha_{s}, \quad b_{4 s}=\sqrt{2}\left(1+2 \alpha_{s}^{2}\right), \\
& b_{5 s}=0, \quad b_{6 s}=\sqrt{\frac{2}{3}} \alpha_{s}\left(1+4 \alpha_{s}^{2}\right), \quad b_{7 s}=0, \quad b_{8 s}=0, \\
& b_{9 s}=-\frac{1-2 \alpha_{s}^{2}}{\sqrt{6} \alpha_{s}}, \quad b_{10 s}=2 \sqrt{2} \alpha_{s}^{2}, \quad b_{11 s}=0, \quad b_{12 s}=0, \\
& c_{1 s}=\sqrt{2} \alpha_{s}, \quad c_{2 s}=0, \quad c_{3 s}=0, \quad c_{4 s}=-\sqrt{\frac{2}{3}}\left(1-2 \alpha_{s}^{2}\right) .
\end{aligned}
$$

We remark that the solution is unique.

We have thus shown that the system (22)-(27), in the limit when terms involving the derivatives with respect to $z$ are neglected, possesses a Hamiltonian structure. This structure consists of the Hamiltonian functional (29) and of a Poisson bracket of the form (31), with $\mathcal{A}^{i j}{ }_{s}=0$, for $i, j=0, \cdots, 5$, and where all the coefficients $W_{k}^{i j}$ are zero, 
except for the following ones:

$$
\begin{aligned}
& W_{k s}^{i 0}=W_{k s}^{0 i}=\delta_{k}^{i}, \quad i=0, \cdots, 5, \\
& W_{0}^{11}{ }_{s}=1, \quad W_{1 s}^{21}=W_{1}^{12}=\sqrt{2}, \quad W_{2 s}^{11}=\sqrt{2}, \quad W_{2}^{31}{ }_{s}=W_{2}^{13}=\sqrt{3}, \\
& W_{3 s}^{21}=W_{3 s}^{12}=\sqrt{3}, \quad W_{3 s}^{51}=W_{3 s}^{15}=2 \alpha_{s}, \quad W_{4 s}^{51}=W_{4 s}^{15}=1, \\
& W_{5}^{31}{ }_{s}=W_{5}^{13}{ }_{s}=2 \alpha_{s}, \quad W_{5}^{41}{ }_{s}=W_{5}^{14}=1, \quad W_{0}^{22}{ }_{s}=1, \quad W_{1 s}^{23}=W_{1 s}^{32}=\sqrt{3}, \\
& W_{2}^{22}{ }_{s}=2 \sqrt{2}, \quad W_{2}^{25}{ }_{s}=W_{2}^{52}{ }_{s}=\sqrt{6} \alpha_{s}, \quad W_{3}^{23}{ }_{s}=W_{3}^{32}{ }_{s}=\sqrt{2}\left(1+2 \alpha_{s}^{2}\right), \\
& W_{3 s}^{24}=W_{3 s}^{42}=\sqrt{2} \alpha_{s}, \quad W_{4 s}^{23}=W_{4 s}^{32}=\sqrt{2} \alpha_{s}, \quad W_{5}^{22}{ }_{s}=\sqrt{6} \alpha_{s}, \\
& W_{5}^{25}{ }_{s}=W_{s_{s}}^{52}=2 \sqrt{2} \alpha_{s}^{2}, \quad W_{0}^{33}=1, \quad W_{1}^{35}{ }_{s}=W_{s_{s}}^{53}=2 \alpha_{s}, \quad W_{2}^{33}=\sqrt{2}\left(1+2 \alpha_{s}^{2}\right), \\
& W_{2 s}^{34}=W_{2}^{43}=\sqrt{2} \alpha_{s}, \quad W_{3 s}^{35}=W_{3 s}^{53}=\sqrt{\frac{2}{3}} \alpha_{s}\left(1+4 \alpha_{s}^{2}\right) \text {, } \\
& W_{4 s}^{35}=W_{4 s}^{53}=-\sqrt{\frac{2}{3}}\left(1-2 \alpha_{s}^{2}\right), \quad W_{5}^{33}{ }_{s}=\sqrt{\frac{2}{3}} \alpha_{s}\left(1+4 \alpha_{s}^{2}\right), \quad W_{5}^{34}{ }_{s}=W_{5}^{43}=-\sqrt{\frac{2}{3}}\left(1-2 \alpha_{s}^{2}\right), \\
& W_{0 s}^{44}=1, \quad W_{1 s}^{45}=W_{1 s}^{54}=1, \quad W_{3 s}^{45}=W_{3 s}^{54}=-\sqrt{\frac{2}{3}}\left(1-2 \alpha_{s}^{2}\right), \\
& W_{4 s}^{45}=W_{4 s}^{54}=-\frac{1-2 \alpha_{s}^{2}}{\sqrt{6} \alpha_{s}}, \quad W_{5 s}^{44}=-\frac{1-2 \alpha_{s}^{2}}{\sqrt{6} \alpha_{s}}, \quad W_{0}^{55}=1, \quad W_{2}^{55}=2 \sqrt{2} \alpha_{s}^{2}, \\
& W_{5}^{55}=-\frac{1+2 \alpha_{s}^{2}-8 \alpha_{s}^{4}}{\sqrt{6} \alpha_{s}} .
\end{aligned}
$$

Now we intend to determine the Hamiltonian structure for the full model (22)-(27), including the terms involving $z$-derivatives. To this scope we follow the procedure of Ref. [29]. According to such procedure, the Hamiltonian functional for the full model corresponds to the one for its perpendicular reduction, that is to the functional (29), in this case. The Poisson bracket for the full model, on the other hand, has the form (30), with the coefficients $W_{k s}^{i j}$ equal to those found for the perpendicular reduction, and with coefficients $\mathcal{A}^{i j}{ }_{s}$ such that the the full model equations are retrieved, as equations of motion, and that the properties of a Poisson bracket are respected. Bilinearity and Leibinz identity are clearly satisfied for any choice of $\mathcal{A}^{i j}{ }_{s}$. On the other hand, antisymmetry amounts to the condition $\mathcal{A}^{i j}{ }_{s}=\mathcal{A}^{j i}{ }_{s}$, whereas the Jacobi identity implies that the coefficients $W_{k s}^{i j}$ and $\mathcal{A}^{i j}{ }_{s}$ must satisfy [29]

$$
W_{r}^{j k}{ }_{s} \mathcal{A}_{s}^{r i}=W_{r}^{k i}{ }_{s} \mathcal{A}^{r j}{ }_{s}=W_{r}^{i j}{ }_{s} \mathcal{A}^{r k}{ }_{s}, \quad i, j, k=0, \cdots, 5,
$$

where the sum over the repeated index $r$ is understood.

With the help of Eq. (34), one obtains that the full model equations (22)-(27) are obtained from the Hamiltonian (29) and from a bracket of the form (30), if

$$
\mathcal{A}^{i j}{ }_{s}=\left(\mathcal{W}^{(1)}{ }_{s}\right)_{i}^{j}
$$


where the expression for $\mathcal{W}_{s}^{(1)}$ is given in Eq. (35). The matrix $\mathcal{W}^{(1)}{ }_{s}$ is symmetric, which guarantees antisymmetry of the Poisson bracket.

Concerning the Jacobi identity, we note that, for any fixed $i$, one has

$$
W_{r}^{k i}{ }_{s} \mathcal{A}^{r j}{ }_{s}=\left(\mathcal{W}^{(i)}{ }_{s}\right)_{r}^{k}\left(\mathcal{W}^{(1)}{ }_{s}\right)_{j}^{r}=\left(\mathcal{W}^{(i)}{ }_{s}\right)_{r}^{j}\left(\mathcal{W}^{(1)}{ }_{s}\right)_{k}^{r}=W_{r}^{i j}{ }_{s} \mathcal{A}^{r k}{ }_{s},
$$

which gives the second equality in Eq. (41). In the second step of Eq. (43) we made use of the fact that the matrix $\left(\mathcal{W}^{(i)} s\right)_{r}^{k}\left(\mathcal{W}^{(1)} s\right)_{j}^{r}$ is symmetric, given that all the matrices $W^{(j)}$ s are symmetric and pairwise commute. Similarly, one has

$$
W_{r}^{i j}{ }_{s} \mathcal{A}^{r k}{ }_{s}=\left(\mathcal{W}^{(j)}{ }_{s}\right)_{r}^{i}\left(\mathcal{W}^{(1)}{ }_{s}\right)_{k}^{r}=\left(\mathcal{W}^{(j)}{ }_{s}\right)_{r}^{k}\left(\mathcal{W}^{(1)}{ }_{s}\right)_{i}^{r}=W_{r}^{j k}{ }_{s} \mathcal{A}^{r i}{ }_{s}
$$

which proves the remaining equality in Eq. (41). The Jacobi identity is thus satisfied.

To summarize, we have shown that the model (22)-(27) admits a Hamiltonian structure. The Hamiltonian functional is given by the functional (29). The Poisson bracket is given by Eq. (30), with coefficients $W_{k}^{i j}$ equal to zero except for those indicated in Eq. (40), and coefficients $\mathcal{A}^{i j}{ }_{s}$ given by Eq. (42).

We find it useful, at this point, in order also to help the contact with the existing literature, to reformulate the model equations (22)-(27), in terms of the dimensional physical variables introduced in Eq. (14). In terms of such variables the model can be written as

$$
\begin{array}{r}
\frac{\partial n_{s}}{\partial t}=-\frac{c}{B}\left[\phi, n_{s}\right]+n_{0} \nabla_{\|} u_{s} \\
\frac{\partial}{\partial t}\left(u_{s}+\frac{q_{s}}{m_{s} c} A\right)=-\frac{c}{B}\left[\phi, u_{s}+\frac{q_{s}}{m_{s} c} A\right] \\
+\frac{T_{s}}{m_{s}} \nabla_{\|}\left(\frac{T_{\|_{s}}}{T_{s}}+\frac{n_{s}}{n_{0}}\right)-\frac{q_{s}}{m_{s}} \frac{\partial \phi}{\partial z} \\
\frac{\partial T_{\|_{s}}}{\partial t}=-\frac{c}{B}\left[\phi, T_{\|_{s}}\right]+\frac{2}{n_{0}} \nabla_{\|}\left(q_{\|_{s}}+n_{0} T_{s} u_{s}\right) \\
\frac{\partial T_{\perp s}}{\partial t}=-\frac{c}{B}\left[\phi, q_{\|_{s}}\right]-v_{t s} \nabla_{\|}\left(\sqrt{6} \alpha_{s} q_{\perp_{s}}-\frac{3}{2} n_{0} v_{t s} T_{\|_{s}}\right) \\
\frac{\partial q_{\perp s}}{\partial t}=-\frac{1}{n_{0}} \nabla_{\|} q_{\perp s} \\
B
\end{array}
$$

where $\nabla_{\|} f=(1 / B)[A, f]-\partial_{z} f$, for a generic function $f$. Such operator represents a normalized gradient along the magnetic field $\mathbf{B}=\nabla A \times \hat{z}+B \hat{z}$.

Eqs. (45) and (46) express particles number and canonical momentum conservation. Eqs. (47)-(48) govern the evolution of the temperature and heat flux fluctuations in the direction parallel to the constant component of the 
magnetic field, and similarly (49) and (50) determine the evolution of temperature and heat flux fluctuations due to the motion perpendicular to the magnetic guide field.

Each dynamical field undergoes the advection by the $\mathbf{E} \times \mathbf{B}$ velocity, represented by the first term on the righthand side of each equations, where the electrostatic potential $\phi$ appears. In addition to this effect, each field evolves according to the gradients of neighbouring moments, that form along the magnetic field. For instance, temperature evolutions are affected by the presence of velocity and heat flux gradients. On the other hand, parallel/perpendicular heat fluxes are affected by parallel/perpendicular temperature gradients but also by perpendicular/parallel heat fluxes, through the coupling related to the coefficients $\alpha_{s}$.

From the inspection of the expression for the coefficients $W_{4}^{45}{ }_{s}, W_{4}^{54}{ }_{s}, W_{5}^{44}{ }_{s}, W_{5}^{55}{ }_{s}$ in Eq. (40) it emerges that, in the Hamiltonian model, the constants $\alpha_{s}$ cannot be zero. Therefore, the existence of a Hamiltonian structure for the model, with a Poisson bracket of the form (30), requires the existence of the terms in Eqs. (25) and (27), which introduce a direct coupling between the parallel and perpendicular heat fluxes. Although, to the best of our knowledge, its Hamiltonian structure is not known, the model in the case $\alpha_{s}=0$, is nevertheless energy conserving.

\section{NORMAL FIELDS AND CASIMIR INVARIANTS}

In this section we show that there exists an alternative set of dynamical variables, in terms of which the model (22)-(27) takes a more compact form, unveiling the existence of some conservation laws. In particular, the Poisson bracket in terms of such new variables takes a remarkably simpler form, which greatly simplifies the identification of Casimir invariants.

In order to identify the new appropriate set of dynamical variables, it is important to note that the generic equation of the model can be written as

$$
\begin{aligned}
& \frac{\partial g_{m_{s}}}{\partial t}=-\frac{c}{B}\left[\phi, g_{m_{s}}\right]+\frac{v_{t s}}{B}\left(\mathcal{W}_{s}^{(1)}\right)^{m n}\left[A, g_{n_{s}}\right]-v_{t s}\left(\mathcal{W}^{(1)}\right)^{m n} \frac{\partial}{\partial z} g_{n_{s}} \\
& -\delta_{m 1} v_{t s} \frac{q_{s}}{T_{s}} \frac{\partial \phi}{\partial z}+\sqrt{m !}\left(\delta_{m 0}+\delta_{m 2}\right) \frac{v_{t s}^{2}}{c} \frac{q_{s}}{T_{s}} \frac{\partial A}{\partial z}, \quad 0 \leq m \leq 5 .
\end{aligned}
$$

The matrices $\mathcal{W}^{(1)} s$ are real and symmetric, therefore each of such matrices possesses six real eigenvalues $\lambda_{0 s}, \lambda_{1 s} \cdots, \lambda_{5 s}$. Moreover, for each species $s$, there exists an orthogonal matrix $U_{s}$, such that $U_{s}{ }^{T} \mathcal{W}^{(1)}{ }_{s} U_{s}=\Lambda_{s}$ where $\Lambda_{s}=\operatorname{diag}\left(\lambda_{0 s}, \lambda_{1 s}, \cdots, \lambda_{5 s}\right)$. If we perform the change of variables $\left(g_{0_{s}}, g_{1_{s}}, \cdots, g_{5 s}\right) \rightarrow\left(G_{0 s}, G_{1 s}, \cdots, G_{5 s}\right)$, where

$$
G_{i s}=U_{s}^{T^{i m}} G_{m s}
$$


the model equations (51) transform into

$$
\begin{aligned}
& \frac{\partial G_{i s}}{\partial t}=-\frac{c}{B}\left[\phi-\lambda_{i s} \frac{v_{t s}}{c} A, G_{i s}\right]-v_{t s} \lambda_{i s} \frac{\partial G_{i s}}{\partial z} \\
& -v_{t s} \sqrt{m !} U_{s}^{T^{i m}}\left(\delta_{m 1} \frac{q_{s}}{T_{s}} \frac{\partial \phi}{\partial z}-v_{t s}\left(\delta_{m 0}+\delta_{m 2}\right) \frac{q_{s}}{T_{s}} \frac{\partial A}{\partial z}\right), \quad i=0, \cdots, 5
\end{aligned}
$$

The form (53) for the model equations presents some advantages with respect to the original formulation (51). Indeed, the evolution equation for each $G_{i s}$ depends only on $G_{i s}$ (apart from the implicit dependence on the other dynamical variables coming from the potentials $\phi$ and $A$ ), whereas the evolution equations for $g_{i_{s}}$ in general involved explicitly also the dynamical variables $g_{i-1_{s}}$ and $g_{i+1_{s}}$. Also, the form of Eq. (53), in the two-dimensional limit assuming $z$ as ignorable coordinate, reveals that the fields $G_{i s}$ are Lagrangian invariants. Indeed, in the two-dimensional limit, the generic equations (53) can be written as

$$
\frac{\partial G_{i s}}{\partial t}+\mathbf{v}_{i s} \cdot \nabla G_{i s}=0
$$

where

$$
\mathbf{v}_{i s}=\frac{c}{B} \hat{z} \times \nabla\left(\phi-\lambda_{i s} \frac{v_{t s}}{c} A\right)
$$

is an incompressible velocity field advecting the dynamical field $G_{i s}$. The two contributions in such velocity field are related to the $\mathbf{E} \times \mathbf{B}$ velocity field and to the free streaming along the "poloidal" magnetic field, respectively, in the original drift-kinetic description. The existence of such advecting field becomes transparent when the model is formulated in terms of the $G_{i s}$ variables.

The explicit expressions for the eigenvalues $\lambda_{0 s}, \cdots, \lambda_{5 s}$ can be found in A. The columns $v_{s}$ of the matrices $U_{s}$, on the other hand, can be found from $\mathcal{W}^{(1)}{ }_{s} v_{s}=\Lambda_{s} v_{s}$

For the sake of our analysis, we point out that the first three rows of the matrices $U_{s}$ have the following structure:

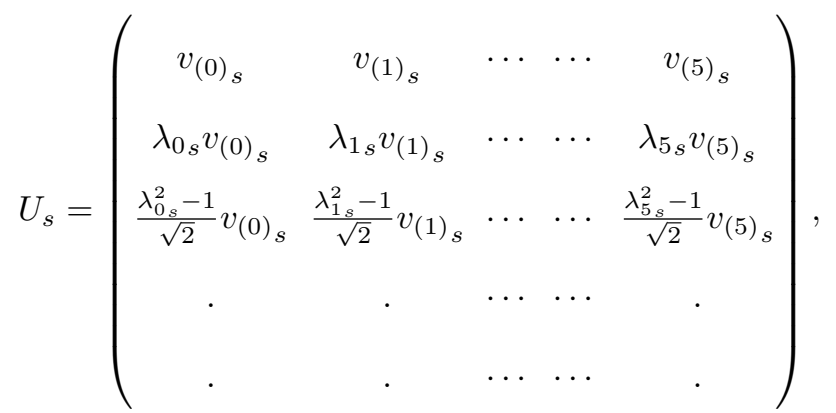

where $v_{(0)_{s}}, \cdots, v_{(5)}$ are constants determined by the normalization condition. By virtue of the expression (56), the 
model equations (53) can also be written as

$$
\begin{aligned}
& \frac{\partial G_{i s}}{\partial t}=-\frac{c}{B}\left[\phi-\lambda_{i s} \frac{v_{t s}}{c} A, G_{i s}\right]-v_{t s} \lambda_{i s} \frac{\partial G_{i s}}{\partial z} \\
& -\lambda_{i s} v_{t s} v_{(i)} \frac{q_{s}}{T_{s}} \frac{\partial}{\partial z}\left(\phi-\lambda_{i s} \frac{v_{t s}}{c} A\right), \quad i=0, \cdots, 5 .
\end{aligned}
$$

The Hamiltonian functional (29), in terms of the new variables, reads

$$
\begin{aligned}
& \bar{H}\left(G_{01}, \cdots, G_{5 \mathcal{N}}\right)=\frac{1}{2} \sum_{s} \int d^{3} x\left[n_{0} T_{s} \sum_{i=0}^{5} G_{i s}^{2}\right. \\
& \left.+q_{s} n_{0} U^{0 l}{ }_{s} G_{l s} \phi-q_{s} n_{0} \frac{v_{t s}}{c} U^{1 l}{ }_{s} G_{l s} A\right],
\end{aligned}
$$

and consequently

$$
\bar{H}_{i s}=n_{0} T_{s} G_{i s}+q_{s} n_{0} v_{(i)_{s}}\left(\phi-\lambda_{i s} \frac{v_{t s}}{c} A\right)
$$

The advection form of Eq. (57), and the expression for the functional derivatives (59) suggests that, in terms of the new variables, the Poisson bracket for the model is given by

$$
\{F, G\}=\sum_{s} \sum_{i=0}^{5}\left[-\frac{c}{q_{s} B n_{0} v_{(i)} s} \int d^{3} x G_{i_{s}}\left[\frac{\delta F}{\delta G_{i_{s}}}, \frac{\delta G}{\delta G_{i_{s}}}\right]-\lambda_{i_{s}} \frac{v_{t s}}{n_{0} T_{s}} \int d^{3} x \frac{\delta F}{\delta G_{i s}} \frac{\partial}{\partial z} \frac{\delta G}{\delta G_{i_{s}}}\right] .
$$

It is straightforward to verify, for instance by using the procedure adopted in Sec. IV, that the bracket (60) is a Poisson bracket and that in particular it satisfies the Jacobi identity. The bracket (60) has a direct sum structure, consisting of a linear combination of Poisson brackets labelled by the indices $i$ and $s$. Poisson brackets with such a structure are common in reduced fluid models for plasmas and further examples can be found for instance in Refs. $[28,31,38]$. By direct substitution into the expression $\partial_{t} G_{i_{s}}=\left\{G_{i_{s}}, \bar{H}\right\}$, using the bracket $(60)$ and the Hamiltonian (58), it is easy to see that the model equations (57) are retrieved.

As above noticed, the alternative set of variables $G_{0 s}, \cdots, G_{5 s}$ provides a considerable simplification the structure of the Poisson bracket, with respect to the Poisson bracket introduced in Sec. IV in terms of the variables $g_{0}, \cdots, g_{5}$. We refer to such variables, reducing a Poisson bracket to its simplest form, as to normal fields. The main advantage of such simplification is that, from Eq. (60), it is staightforward to identify Casimir invariants for the model. We recall that, Casimir invariants of a Poisson bracket $\{$,$\} (see, e.g., Refs. [25, 26]) are functionals C$, such that

$$
\{C, F\}=0
$$

for any functional F. Because Casimirs commute in particular with the Hamiltonian functional, they are also conserved by the dynamics. 
From the definition (61), it is easy to see that, for the bracket (60), Casimirs are given by

$$
C_{i s}=\int d^{3} x G_{i s}, \quad i=0, \cdots, 5
$$

The conservation of such integrals therefore constraints the dynamics of the model. In particular, in the twodimensional limit, with $z$ as ignorable coordinate, Casimirs correspond to

$$
C_{i s}=\int d^{3} x \mathcal{C}_{i s}\left(G_{i s}\right), \quad i=0, \cdots, 5
$$

where $\mathcal{C}_{i s}$ are arbitrary functions. Therefore, in the presence of such spatial invariance of the system (which is a sensible approximation for laboratory and solar plasmas where a strong component of the magnetic field makes the dynamics independent, at the leading order on one coordinate) one obtains that the evolution of the model is constrained by an infinite number of conservation laws. These are associated with the role, discussed in Sec. IV, of the fields $G_{i s}$ as Lagrangian invariants in the two-dimensional limit.

We remark that if, for a given species $s$, one has $\alpha_{s}=0$, then the $\mathcal{W}^{(1)}{ }_{s}$ becomes a block diagonal matrix with eigenvalues given by

$$
\begin{array}{r}
\lambda_{0 s}=1, \quad \lambda_{1 s}=-1, \quad \lambda_{2 s}=-\sqrt{3+\sqrt{6}}, \\
\lambda_{3 s}=\sqrt{3+\sqrt{6}}, \quad \lambda_{4 s}=-\sqrt{3-\sqrt{6}}, \quad \lambda_{5 s}=\sqrt{3-\sqrt{6}} .
\end{array}
$$

The corresponding matrix $U_{s}$, on the other hand, reads

$$
U_{s}=\left(\begin{array}{cccccc}
0 & 0 & \frac{-3 \sqrt{3+\sqrt{6}}+\sqrt{6(3+\sqrt{6})}}{3 \sqrt{2}} & \frac{3 \sqrt{3+\sqrt{6}}-\sqrt{6(3+\sqrt{6})}}{3 \sqrt{2}} & \frac{3 \sqrt{3-\sqrt{6}}+\sqrt{6(3-\sqrt{6})}}{3 \sqrt{2}} & \frac{-3 \sqrt{3-\sqrt{6}}-\sqrt{6(3-\sqrt{6})}}{3 \sqrt{2}} \\
0 & 0 & \frac{1}{\sqrt{2}} & \frac{1}{\sqrt{2}} & -\frac{1}{\sqrt{2}} & -\frac{1}{\sqrt{2}} \\
0 & 0 & -\sqrt{\frac{3+\sqrt{6}}{6}} & \sqrt{\frac{3+\sqrt{6}}{6}} & -\sqrt{\frac{3-\sqrt{6}}{6}} & \sqrt{\frac{3-\sqrt{6}}{6}} \\
0 & 0 & \frac{1}{\sqrt{2}} & \frac{1}{\sqrt{2}} & 0 & \frac{1}{\sqrt{2}} \\
\frac{1}{\sqrt{2}}-\frac{1}{\sqrt{2}} & 0 & 0 & 0 & 0 \\
\frac{1}{\sqrt{2}} & \frac{1}{\sqrt{2}} & 0 & 0 & 0
\end{array}\right) .
$$

From the expression (64) one sees that, in this case, $v_{(0)}=v_{(1)}=0$. Consequently, the Poisson bracket (60), in this case does not exist, because $v_{(0)_{s}}$ and $v_{(1)_{s}}$ would vanish at the denominator. Thus, in spite of the fact that, also for $\alpha_{s}=0$, the system can be cast in the form (57), a Hamiltonian formulation with Hamiltonian functional given by (59) and Poisson bracket of the form (60) is not possible, thus confirming the conclusions drawn in Sec. IV with regard to the case $\alpha_{s}=0$. 


\section{CONCLUSIONS}

We have presented a Hamiltonian anisotropic electromagnetic fluid model evolving six field variables for each particle species, accounting for the evolution of density, canonical fluid momentum, as well as parallel and perpendicular temperature and heat flux fluctuations. The model is derived by taking moments with respect to Hermite-Laguerre polynomials of a generalized distribution function and imposing as closure a proportionality relation between the moments $g_{40_{s}}$ and $g_{21_{s}}$ and the perpendicular and parallel heat flux fluctuations, respectively. Such closure amounts to introduce terms coupling directly the evolutions of the parallel and perpendicular heat fluxes, for each species.

As Hamiltonian for the fluid model a conserved functional obtained self-consistently form the Hamiltonian of the parent drift-kinetic model is assumed. The Poisson bracket is then constructed by assuming it to possess the form of a Lie-Poisson bracket, which is very common for reduced fluid plasma models. A general form of bilinear functional satisfying the Leibniz condition and yielding, from the assumed Hamiltonian, the model equations in 2D, is considered. By imposing the additional constraints of antisymmetry and the Jacobi identity the expression for the Lie-Poisson bracket of the model in the $2 \mathrm{D}$ limit is thus obtained. The extension to $3 \mathrm{D}$ is then carried out following a procedure adopted in Ref. [29]. From the expression of the Poisson bracket it emerges that the proportionality coefficients $\alpha_{s}$ in the closure relation cannot be zero, thus implying the presence of the terms coupling parallel and perpendicular heat fluxes in the model equations, in order to assure a Hamiltonian structure with a Lie-Poisson bracket.

An alternative set of dynamical variables (normal fields) is then introduced, in terms of which the Poisson bracket of the model simplifies considerably. This made the identification of Casimir invariants particularly simple. In the $2 \mathrm{D}$ limit, $6 \mathcal{N}$ infinite families of Casimir invariants are found. These express the property of the normal fields of being Lagrangian invariants of the model. This unveils a remarkable feature of the model in the 2D limit: the model can indeed be reformulated as a system of $6 \mathcal{N}$ advection equations for $6 \mathcal{N}$ Lagrangian invariants transported by incompressible generalized velocity fields. Such velocity fields, corresponding to those indicated in Eqs. (55), are associated with stream functions corresponding to linear combinations of electrostatic and magnetic potential. These account for the $\mathbf{E} \times \mathbf{B}$ velocity and free stream along the fluctuating part of the magnetic field. A similar feature occurs in other Hamiltonian reduced plasma models, e.g. in Refs. [24, 28, 31, 38] but none of such models accounted also for perpendicular temperature and heat fluxes.

Finally, it is worth emphasizing a remark about the adopted closure in the case $\alpha_{s}=0$. This corresponds to 
eliminating the terms directly coupling parallel and perpendicular heat fluxes for a given species and is the closure that appears to be adopted in the models of Refs. [2, 7], when taken in the limit of negligible finite Larmor radus effects, background inhomogeneities and dissipation. The Hamiltonian formulation found for the present model fails in the case $\alpha_{s}=0$. This special case turns out to be energy conserving but no Poisson bracket of the form (30) is able to yield the model equations when combined with the assumed Hamiltonian functional. This suggests that, for the particular closure $\alpha_{s}=0$, the Hamiltonian structure requires a different form of Poisson bracket or that ingredients neglected in the present analysis (e.g. finite Larmor radius effects) are needed to restore a Hamiltonian structure with the adopted form of Poisson bracket.

The author acknowledges useful discussions with the Nonlinear Dynamics Team of the Centre de Physique Théorique. Financial support was received from the CNRS through the PEPS project GEOPLASMA2.

\section{Appendix A: Determining the eigenvalues of the matrices $\mathcal{W}^{(1)}{ }_{s}$}

For a fixed species, the eigenvalues of the matrix $\mathcal{W}^{(1)}{ }_{s}$ correspond to the roots of the characteristic polynomial

$$
\lambda^{6}-\left(7+4 \alpha_{s}^{2}\right) \lambda^{4}+\left(9+12 \alpha_{s}^{2}\right) \lambda^{2}-3
$$

which we can rewrite as

$$
u^{3}-\left(7+4 \alpha_{s}^{2}\right) u^{2}+\left(9+12 \alpha_{s}^{2}\right) u-3
$$

where $u=\lambda^{2}$.

Following a standard procedure to determine the roots of an algebraic equation of third degree, we operate the transformation $u=y+\left(7+4 \alpha_{s}^{2}\right) / 3$, which casts the polynomial (A2) in the form

$$
y^{3}-\frac{16 \alpha_{s}^{4}+20 \alpha_{s}^{2}+22}{3} y-\frac{32 \alpha_{s}^{2}+80 \alpha_{s}^{4}}{9}-\frac{128 \alpha_{s}^{6}+200}{27} .
$$


The roots of (A3) are then given by

$$
\begin{array}{r}
y_{1}=\frac{2}{3} \sqrt{2} \sqrt{8 \alpha_{s}^{4}+10 \alpha_{s}^{2}+11} \cos \left[\frac { 1 } { 3 } \operatorname { A r g } \left(\frac{4}{27}\left(16 \alpha_{s}^{6}+30 \alpha_{s}^{4}+12 \alpha_{s}^{2}+25\right)\right.\right. \\
\left.\left.+\frac{2}{3} i \sqrt{2} \sqrt{24 \alpha_{s}^{8}+40 \alpha_{s}^{6}+36 \alpha_{s}^{4}+30 \alpha_{s}^{2}+1}\right)\right] \\
y_{2}=\frac{2}{3} \sqrt{2} \sqrt{8 \alpha_{s}^{4}+10 \alpha_{s}^{2}+11} \cos \left[\frac { 1 } { 3 } \operatorname { A r g } \left(\frac{4}{27}\left(16 \alpha_{s}^{6}+30 \alpha_{s}^{4}+12 \alpha_{s}^{2}+25\right)\right.\right. \\
\left.\left.+\frac{2}{3} i \sqrt{2} \sqrt{24 \alpha_{s}^{8}+40 \alpha_{s}^{6}+36 \alpha_{s}^{4}+30 \alpha_{s}^{2}+1}+2 \pi\right)\right] \\
y_{3}=\frac{2}{3} \sqrt{2} \sqrt{8 \alpha_{s}^{4}+10 \alpha_{s}^{2}+11} \cos \left[\frac { 1 } { 3 } \operatorname { A r g } \left(\frac{4}{27}\left(16 \alpha_{s}^{6}+30 \alpha_{s}^{4}+12 \alpha_{s}^{2}+25\right)\right.\right. \\
\left.\left.\left.+\frac{2}{3} i \sqrt{2} \sqrt{24 \alpha_{s}^{8}+40 \alpha_{s}^{6}+36 \alpha_{s}^{4}+30 \alpha_{s}^{2}+1}\right)+4 \pi\right)\right]
\end{array}
$$

and the eigenvalues of the matrix $\mathcal{W}^{(1)}{ }_{s}$ correspond then to

$$
\begin{aligned}
& \lambda_{0,1_{s}}= \pm \sqrt{y_{1}+\frac{7+4 \alpha_{s}^{2}}{3}}, \\
& \lambda_{2,3_{s}}= \pm \sqrt{y_{2}+\frac{7+4 \alpha_{s}^{2}}{3}}, \\
& \lambda_{4,5_{s}}= \pm \sqrt{y_{3}+\frac{7+4 \alpha_{s}^{2}}{3}} .
\end{aligned}
$$

[1] A. Brizard, Phys. Fluids B 4, (1992) 1213.

[2] B. Scott, Phys. Plasmas 17, (2010) 102306.

[3] T. Passot, P.L. Sulem, Phys. Plasmas 11, (2004) 5173.

[4] T. Passot, P.L. Sulem, Phys. Plasmas 14, (2007) 082502.

[5] T. Passot, P.L. Sulem, P. Hunana, Phys. Plasmas 19, (2012) 082113.

[6] P.B. Snyder, G.W. Hammett, W. Dorland, Phys. Plasmas 4, (1997) 3974.

[7] P.B. Snyder, G.W. Hammett, Phys. Plasmas 8, (2001) 3199.

[8] H. Sugama, T.-H Watanabe, W. Horton, Phys. Plasmas 8, (2001) 2617.

[9] Y. Sarazin, G. Dif-Pradalier, D. Zarzoso, X. Garbet, Ph. Ghendrih, V. Grandgirard, Plasma Phys. Control. Fusion 51, (2009) 115003.

[10] P. J. Morrison, Phys. Lett. 80A, (1980) 383.

[11] J. E. Marsden, A. Weinstein, Physica D 4, (1982) 394.

[12] B.A. Kupershmidt, Ju. I Manin, Funktsional. Anal. i Prilozhen. 1, (1978) 25.

[13] J. Gibbons, Physica D 3 (1981) 503.

[14] J. Gibbons, D.D. Holm, C. Tronci, Phys. Lett. A 372, (2008) 1024.

[15] J.E. Marsden, P.J. Morrison, A. Weinstein, Contemp. Math. 28, (1984) 115.

[16] A. A. Chesnokov, M.V. Pavlov, Acta Appl. Math. 122 (2012) 367.

[17] M. Perin, C. Chandre, P.J. Morrison, E. Tassi, Annals of Physics 348, (2014) 50.

[18] M. Perin, C. Chandre, P.J. Morrison, E. Tassi, J. Phys. A : Math. Theor. 48, (2015) 275501.

[19] E. Tassi, J. Phys. A: Math. Theor. 47, (2014) 195501.

[20] E. Tassi, Eur. Phys. J. D 68, (2014) 196.

[21] E. Tassi, Journal of Physics: Conference Series 561, (2014) 012018.

[22] E. Tassi, Annals of Physics 362, (2015) 239.

[23] R.D. Hazeltine, J.D. Meiss, Plasma Confinement, Dover, New York (2003).

[24] D. Grasso, E. Tassi, J. Plasma Phys. 81, (2015) 495810501.

[25] P.J. Morrison, Rev. Mod. Phys. 70 (1998) 467.

[26] J.E. Marsden, T.S. Ratiu, Introduction to Mechanics and Symmetry, Springer-Verlag, New York (2010).

[27] H.J. de Blank, Proceedings of the 28th Conference on Contr. Fusion and Plasma Phys., Funchal, 18-22 June 2001, Ed. ECA 25A, (2001) 1709.

[28] F.L. Waelbroeck, E. Tassi, Commun. Nonlinear Sci. Numer. Simulat. 17, (2012) 2171. 
[29] E. Tassi, P.J. Morrison, D. Grasso, F. Pegoraro, Nucl. Fusion 50, (2010) 034007.

[30] E. Tassi, P.J. Morrison, F.L. Waelbroeck, D. Grasso, Plasma Phys. and Contr. Fusion 50, (2008) 085014.

[31] F.L. Waelbroeck, R.D. Hazeltine, P.J. Morrison, Phys. Plasmas 16, (2009) 032109.

[32] F.L. Waelbroeck, P.J. Morrison, W. Horton, Plasma Phys. and Contr. Fusion 46, (2004) 1331.

[33] R.D. Hazeltine, C.T. Hsu, P.J. Morrison, Phys. Fluids 30, (1987) 3204.

[34] D. Dagnelund, V.P. Pavlenko, Physica Scripta 71, (2005) 293.

[35] A. Weinstein, Phys. Fluids 26, (1983) 388.

[36] B.N. Kuvshinov, F. Pegoraro, T.J. Schep, Phys. Lett. A 191, (1994) 296.

[37] J.-L. Thiffeault, P.J. Morrison, Physica D 136 (2000) 205.

[38] E. Cafaro, D. Grasso, F. Pegoraro, F. Porcelli, A. Saluzzi, Phys. Rev. Lett. 80, (1998) 4430. 